\title{
Life-threatening hemobilia caused by hepatic pseudoaneurysm after T-tube choledochostomy: report of a case
}

\author{
Yueh-Tsung Lee1,2, Ho Lin², Kuan-Yung Chen³ , Hurng-Sheng Wu'1, Min-Ho Hwang ${ }^{1}$ and Sheng-Lei Yan*4
}

\begin{abstract}
Background: Hemobilia is a rare but lethal biliary tract complication. There are several causes of hemobilia which might be classified as traumatic or nontraumatic. Hemobilia caused by pseudoaneurysm might result from hepatobiliary surgery or percutaneous interventional hepatobiliary procedures. However, to our knowledge, there are no previous reports pertaining to hemobilia caused by hepatic pseudoaneurysm after T-tube choledochostomy.

Case presentation: A 65-year-old male was admitted to our hospital because of acute calculous cholecystitis and cholangitis. He underwent cholecystectomy, choledocholithotomy via a right upper quadrant laparotomy and a temporary T-tube choledochostomy was created. However, on the 19th day after operation, he suffered from sudden onset of hematemesis and massive fresh blood drainage from the T-tube choledochostomy. Imaging studies confirmed the diagnosis of pseudoaneurysm associated hemobilia. The probable association of T-tube choledochostomy with pseudoaneurysm and hemobilia is also demonstrated. He underwent emergent selective microcoils emobolization to occlude the feeding artery of the pseudoaneurysm.

Conclusions: Pseudoaneurysm associated hemobilia may occur after T-tube choledochostomy. This case also highlights the importance that hemobilia should be highly suspected in a patient presenting with jaundice, right upper quadrant abdominal pain and upper gastrointestinal bleeding after liver or biliary surgery.
\end{abstract}

\section{Background}

Hemobilia is a rare but lethal biliary tract complication [1]. Patients with hemobilia usually present with right upper quadrant abdominal pain, jaundice and upper gastrointestinal (UGI) bleeding [2]. There are several causes of hemobilia which might be classified as traumatic [312] or nontraumatic [12-19]. Hemobilia caused by pseudoaneurysm might be due to abdominal trauma [2], liver and biliary surgery $[8,9,11]$, percutaneous interventional hepatobiliary procedures $[7,10,12,20,21]$, tumor $[16-19,22]$ or other infectious processes such as liver abscess [13], cholecystitis [23], cholangitis [17], and pancreatitis $[5,12,17]$. To our knowledge, there are no previous reports pertaining to hemobilia caused by hepatic pseudoaneurysm after T-tube choledochostomy. We describe here a novel case of hemobilia caused by

* Correspondence: yslcsmu@yahoo.com

4 Division of Gastroenterology, Department of Internal Medicine, Chang-Bing Show Chwan Memorial Hospital, Taiwan, ROC

Full list of author information is available at the end of the article pseudoaneurysm in the proper hepatic artery after Ttube choledochostomy. The probable association of $\mathrm{T}$ tube choledochostomy with pseudoaneurysm and hemobilia is discussed.

\section{Case Presentation}

A 65-year-old male was admitted to our hospital because of right upper quadrant abdominal pain, jaundice and fever for 2 days. He denied history of any systemic disease. He had no history of previous abdominal surgery. At initial presentation, his body temperature was $38.6^{\circ} \mathrm{C}$, blood pressure was $142 / 82 \mathrm{mmHg}$, pulse rate was $92 /$ min, and respiratory rate was $22 / \mathrm{min}$. Physical examination revealed jaundice, and tenderness with light palpation at the right upper quadrant of abdomen. Murphy's sign was positive. There was no palpable mass and Courvoisier's sign was negative. The bowel sound was normal in peristalsis and no abdominal bruit was found on auscultation. 
Laboratory studies demonstrated a white blood count of $10,800 / \mathrm{mm}^{3}$ (normal, 4,500 to $10,000 / \mathrm{mm}^{3}$ ), a hemoglobin level of $15.3 \mathrm{~g} / \mathrm{dL}$ (normal 14-18 g/dL), and a platelet count of $22.3 \times 10^{4} / \mathrm{mm}^{3}$ (normal, 13-40 $\times 10^{4} / \mathrm{mm}^{3}$ ). The liver profile showed total bilirubin $=4.3 \mathrm{mg} / \mathrm{dL}$ (nor$\mathrm{mal}, 0.2-1.2 \mathrm{mg} / \mathrm{dL}$ ), direct bilirubin $=3.2 \mathrm{mg} / \mathrm{dL}$ (nor$\mathrm{mal}, 0-0.4 \mathrm{mg} / \mathrm{dL}$ ), alanine aminotransferase $=108 \mathrm{IU} / \mathrm{L}$ (normal, 4-44 IU/L), aspartate aminotransferase $=103$ $\mathrm{IU} / \mathrm{L}$ (normal, 8-38 IU/L), and alkaline phosphatatse = 327 IU/L (normal, 104-338IU/L). The serum levels of amylase and lipase were $166 \mathrm{U} / \mathrm{L}$ (normal, 43-116 U/L) and $208 \mathrm{U} / \mathrm{L}$ (normal, 13-60 U/L) respectively. Blood cultures were negative for aerobic and anaerobic organisms. Abdominal ultrasonography revealed a distended gallbladder with thickened wall and filled with stones. A small amount of ascites was detected in the Morison's pouch. The common bile duct (CBD) was mildly dilated with some hyperechoic spots, indicating sandy CBD stones.

Because acute calculous cholecystitis and cholangitis were impressed on clinical grounds, he underwent cholecystectomy and choledocholithotomy via a right upper quadrant laparotomy. At operation, an inflamed gallbladder with fibrin coating on the edematous wall was found. The Calot's triangle showed dense adhesion and the CBD was distended. The CBD was explored to remove sandy stones, debris and sticky bile. A temporary T-tube choledochostomy was created and Penrose drains were set in the Morison's pouch.

The patient recovered well after the operation without fever episodes or jaundice. He was discharged on the $14^{\text {th }}$ postoperative days. However, on the $19^{\text {th }}$ day after operation, he suffered from sudden onset of hematemesis and massive fresh blood drainage from the T-tube choledochostomy. Hypovolemic shock developed thereafter. He was sent to emergent department of our institution. Laboratory examination revealed a decreased hemoglobin level of $7.0 \mathrm{~g} / \mathrm{dL}$ and an increased total bilirubin level of $2.1 \mathrm{mg} / \mathrm{dL}$. Because hemobilia was highly suspected a contrast enhanced computed tomography (CT) of the abdomen was arranged emergently, revealing a round and hyperdense lesion abutting the T-tube choledochostomy (Figure 1). The reconstructive CT image further demonstrated a saccular mass protruding from the proper hepatic artery (Figure 2). The angiography disclosed a contrast-filled lesion arising from proper hepatic artery, which suggested a pseudoaneurysm formation (Figure 3). The contrast drained into the T-tube choledochostomy directly (Figure 4), indicating that pseudoaneurysm was the cause of hemobilia in our patient. He underwent emergent selective microcoils embolization to occlude the feeding artery of the pseudoaneurysm and the bleeding ceased thereafter (Figure 5). He recovered unevent-

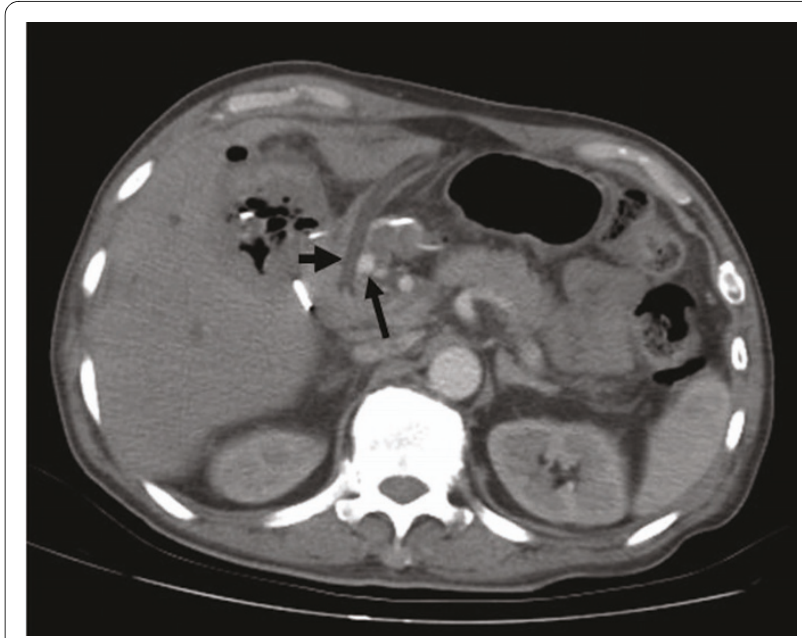

Figure 1 Contrasted enhanced CT of the abdomen was arranged showing a round and hyperdense lesion (long arrow) abutting the T-tube choledochostomy (short arrow).

fully with normal liver profile after a three-month period of follow up.

\section{Conclusions}

Hemobilia, a rare but lethal disaster, was first described by Sandblom in 1948 as the situation of bleeding into the biliary tract following trauma [1]. The classical presentation of hemobilia, also known as Quinckes' triad, includes right upper quadrant abdominal pain, UGI bleeding and jaundice [2]. Blunt and penetrating abdominal trauma, accounting for half of the cases, are the leading causes of hemobilia [2]. Liver and biliary surgery $[4,8,9,11]$, percutaneous liver biopsy $[4,12]$, percutaneous liver tumor ablation [21], and percutaneous transhepatic biliary

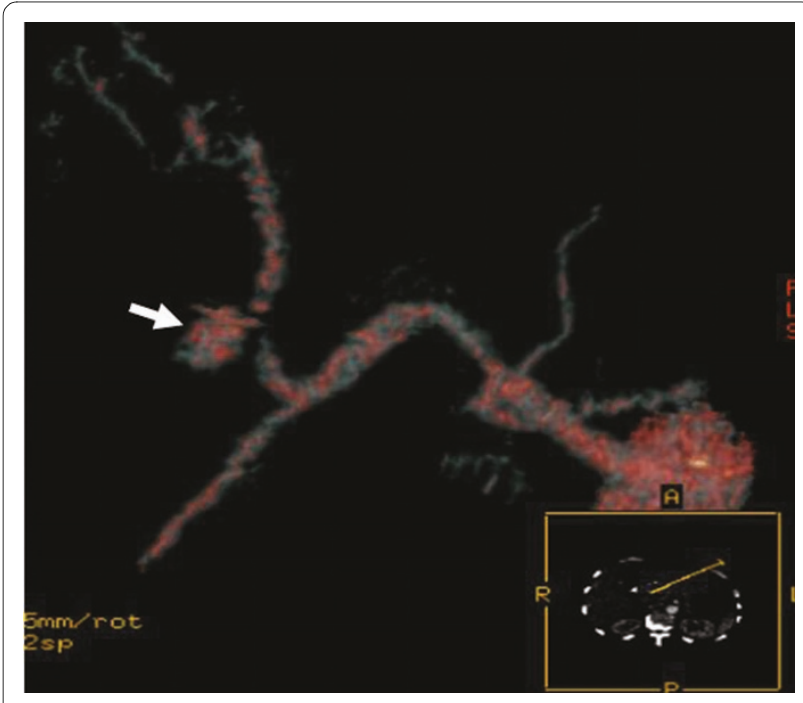

Figure 2 Reconstructive CT image showing a saccular mass protruding from the proper hepatic artery. 


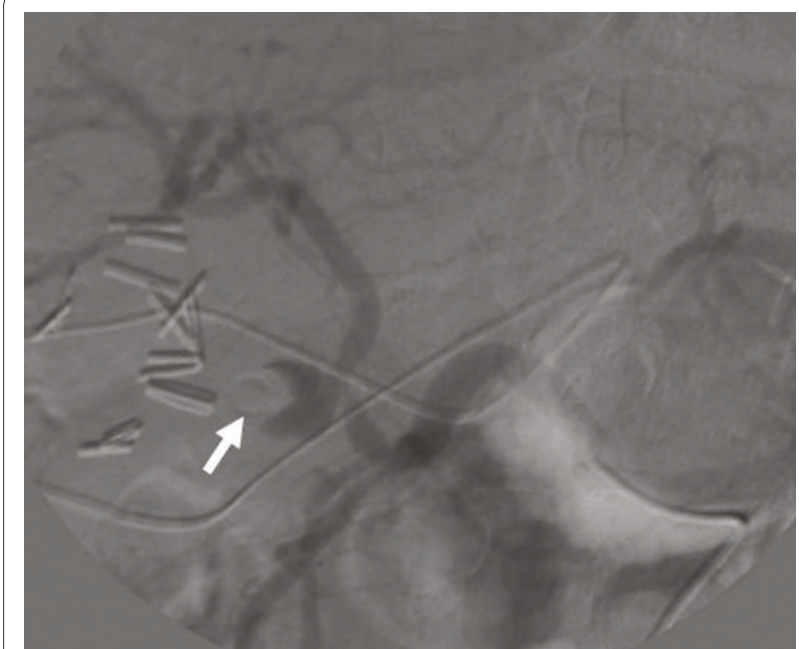

Figure 3 Transarterial angiographic view showing a contrastfilled lesion arising from proper hepatic artery. Adjacent to the contrast-filled lesion was the T-tube (arrow).

drainage and stent placement $[4,7,10,20]$ contribute to the other causes of traumatic hemobilia. Nontraumatic causes of hemobilia include aneurysm or pseudoaneurysm caused by liver abscess [13], choledocholithiasis and related infection $[14,23]$, pancreatitis $[5,12,15,16,18]$, and tumor [16-19,22]. However, to the best of our knowledge, there are no previous reports in the English literature regarding hepatic pseudoaneurysm associated hemobilia after T-tube choledochostomy. This unusual case could be added to the causal list of hemobilia after surgery.

As for the etiological cause of pseudoaneurysm formation in our case, we propose that mechanical compression of the T-tube on the bile duct mucosa and adjacent proper hepatic artery might have caused erosion of the

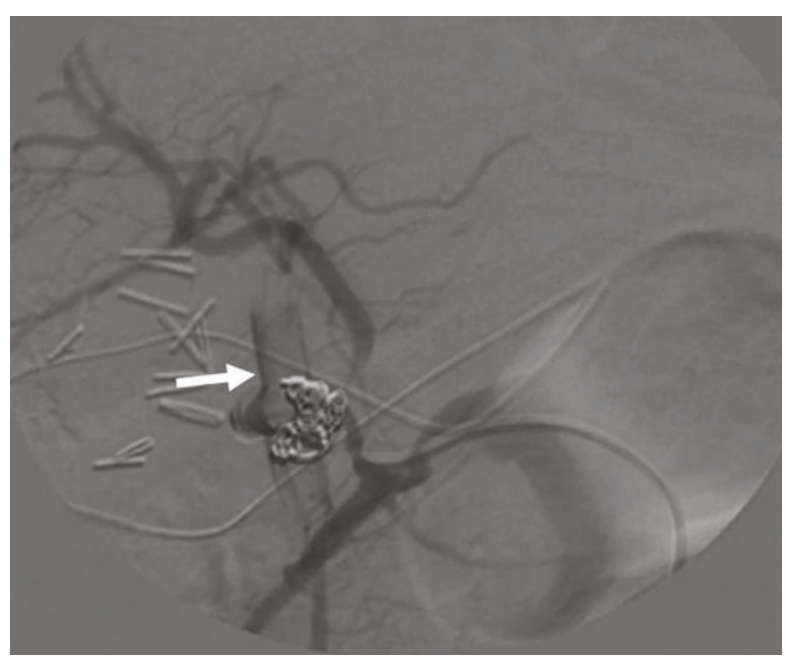

Figure 4 Transarterial angiographic view showing that contrast drained into the T-tube choledochostomy (arrow)

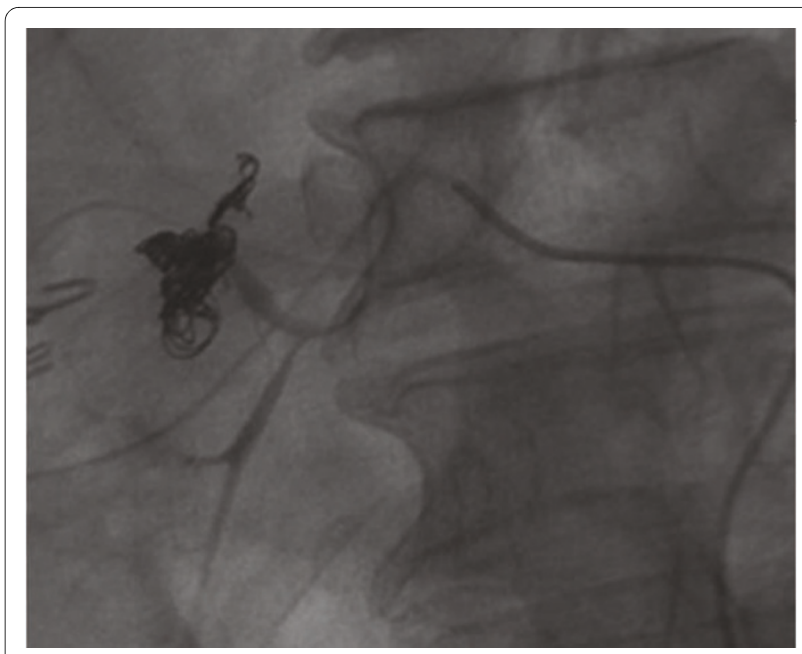

Figure 5 Transarterial angiographic view showing that the pseudoaneurym was occluded using selective microcoils emobolization.

blood vessel, and induced pseudoaneurysm formation. There are evidences that might support our proposal. Firstly, anatomically speaking, the common bile duct runs along with the proper hepatic artery within the hepatoduodenal ligament. Secondly, the contrast-filled hyperdense pseudoaneurysm found at abdominal CT was just close to the T-tube. The angiographic features further disclosed that the lunar pseudoaneurysm was compressed by the T-tube and the contrast flew directly from the proper hepatic artery into the T-tube.

The diagnosis of hemobilia can be confirmed by endoscopy examination or endoscopic retrograde cholangiopancreatography to directly observe bleeding from the ampulla of Vater [12]. Doppler ultrasound can be used to detect the pulsatile mass with blood flow into the biliary tracts [24]. Tumors, abscesses, and the location of aneurysms and pseudoaneurysms can be identified by abdominal CT [25]. Furthermore, contrast-enhanced magnetic resonance imaging can be used to demonstrate the correlation of blood flow with bile ducts to support the diagnosis of hemobilia [26]. However, transarterial angiography should be considered as the imaging modality of choice, because it can be used to detect and embolize the bleeders $[2,9,22,27,28]$. Surgical ligation, excision of aneurysm and pseudoaneurysm or partial hepatectomy is only conserved for failed embolization or tumor-related hemobilia [2,22]. Furthermore, recent articles suggest that minimally invasive procedures were offered for treatment of hemobilia, especially for tumor associated hemobilia $[18,19,22]$.

In conclusion, pseudoaneurym associated hemobilia may occur after T-tube choledochostomy. This case also highlights the importance that hemobilia should be highly suspected in a patient presenting with jaundice, 
right upper quadrant abdominal pain and UGI bleeding after liver and biliary surgery. Once a diagnosis is established, angiographic intervention should be performed without delay because it is a life-threatening situation.

\section{Consent}

Written informed consent was obtained from the patient for publication of this case report and any accompanying images. A copy of the written consent is available for review by the Editor-in-Chief of this journal.

\section{Abbreviations \\ UGl: upper gastrointestinal; CT: computed tomography; CBD: common bile duct. \\ Competing interests \\ The authors declare that they have no competing interests. \\ Authors' contributions \\ All authors have read and approved the final version of manuscript. LYT: surgi- cal operation, drafting the manuscript; LH: helped to draft and edit the manu- script; CKY: carried out radiological intervention; WHS: involved in the clinical management of the patient; $\mathrm{HMH}$ : revised the manuscript critically and added substantial intellectual content; YSL: final review, supervision of scientific con- tent of manuscript.}

\section{Author Details}

1Department of Surgery, Chang-Bing Show Chwan Memorial Hospital, Changhua County, Taiwan, ROC, ${ }^{2}$ Department of Life Sciences, Nationa Chung-Hsing University, Taichung City, Taiwan, ROC, ${ }^{3}$ Department of Radiology, Chang-Bing Show Chwan Memorial Hospital, Changhua County, Taiwan and ${ }^{4}$ Division of Gastroenterology, Department of Internal Medicine, Chang-Bing Show Chwan Memorial Hospital, Taiwan, ROC

Received: 19 January 2010 Accepted: 14 July 2010

Published: 14 July 2010

\section{References}

1. Sandblom P: Hemorrhage into the biliary tract following trauma; traumatic hemobilia. Surgery 1948, 24:571-586.

2. Green $M H$, Duell RM, Johnson CD, Jamieson NV: Haemobilia. Br J Surg 2001, 88:773-786.

3. Lygidakis NJ, Okazaki M, Damtsios G: latrogenic hemobilia: how to approach it. Hepatogastroenterology 1991, 38:454-457.

4. Curet P, Baumer R, Roche A, Grellet J, Mercadier M: Hepatic hemobilia of traumatic or iatrogenic origin: recent advances in diagnosis and therapy, review of the literature from 1976 to 1981 . World J Surg 1984 8:2-8.

5. de Perrot M, Berney T, Buhler L, Delgadillo X, Mentha G, Morel P. Management of bleeding pseudoaneurysms in patients with pancreatitis. Br J Surg 1999, 86:29-32.

6. Messina LM, Shanley CJ: Visceral artery aneurysms. Surg Clin North Am 1997, 77:425-442.

7. Rai R, Rose J, Manas D: Potentially fatal haemobilia due to inappropriate use of an expanding biliary stent. World J Gastroenterol 2003, 9:2377-2378.

8. England RE, Marsh PJ, Ashleigh R, Martin DF: Case report: pseudoaneurysm of the cystic artery: a rare cause of haemobilia. Clin Radiol 1998, 53:72-75

9. Napolitano V, Cirocchi R, Spizzirri A, Cattorini L, La Mura F, Farinella E, Morelli U, Migliaccio C, Del Monaco P, Trastulli S, Di Patrizi MS, Milani D, Sciannameo F: A severe case of hemobilia and biliary fistula following an open urgent cholecystectomy. World J Emerg Surg 2009, 4:37.

10. Layec S, D'Halluin PN, Pagenault M, Bretagne JF: Massive hemobilia during extraction of a covered self-expandable metal stent in a patient with portal hypertensive biliopathy. Gastrointest Endosc 2009, 70:555-556.
11. Facy O, Angot C, Fortier S, Guiu B, Krause D, Ortega-Deballon P: Pseudoaneurysm of the cystic artery: A life-threatening cause of hemobilia after cholecystectomy. Surgery 2009 in press.

12. Hendriks MP, Wanten GJ, Drenth JP: Management of hemobilia and pancreatitis after liver biopsy: a key role for endoscopic retrograde cholangiopancreaticography. Liver Transp/ 2009, 15:1653-1654.

13. Liou TC, Ling CC, Pang KK: Liver abscess concomitant with hemobilia due to rupture of hepatic artery aneurysm: a case report. Hepatogastroenterology 1996, 43:241-244.

14. Kaman L, Kumar S, Behera A, Katariya RN: Pseudoaneurysm of the cystic artery: a rare cause of hemobilia. Am J Gastroenterol 1998, 93:1535-1537.

15. Liu TT, Hou MC, Lin HC, Chang FY, Lee SD: Life-threatening hemobilia caused by hepatic artery pseudoaneurysm: a rare complication of chronic cholangitis. World J Gastroenterol 2003, 9:2883-2884

16. Manolakis AC, Kapsoritakis AN, Tsikouras AD, Tsiopoulos FD, Psychos AK Potamianos SP: Hemobilia as the initial manifestation of cholangiocarcinoma in a hemophilia B patient. World J Gastroenterol 2008, 14:4241-4244

17. Kim JD, Lee KM, Chung WC, Chang UI, Lee BI, Lee JS, Kim HJ, Yang JM, Choi KY, Chung IS: Acute pancreatitis and cholangitis caused by hemobilia from biliary papillomatosis. Gastrointest Endosc 2007, 65:177-180.

18. Paikos D, Katsinelos P, Kontodimou K, Chatzimavroudis G, Pilpilidis I, Moschos I, Soufleris K, Papaziogas B: Acute recurrent pancreatitis complicating hemobilia in a patient with hepatocellular cancer and recipient of anticoagulants successful treatment with metal stent placement (pancreatitis complicating hemobilia). Pancreas 2007, 34:168-169.

19. Rerknimitr R, Kongkam $P$, Kullavanijaya P: Treatment of tumor associated hemobilia with a partially covered metallic stent. Endoscopy 2007 39(Suppl 1):E225

20. Cho YD, Cheon YK, Moon JH, Jeong SW, Jang JY, Lee JS, Shim CS: Clinical role of frequency-doubled double-pulsed yttrium aluminum garnet laser technology for removing difficult bile duct stones (with videos). Gastrointest Endosc 2009, 70:684-689.

21. Goto E, Tateishi R, Shiina S, Masuzaki R, Enooku K, Sato T, Ohki T, Kondo Y, Goto T, Yoshida H, Omata M: Hemorrhagic Complications of Percutaneous Radiofrequency Ablation for Liver Tumors. J Clin Gastroenterol 2009 in press.

22. Takao Y, Yoshida H, Mamada Y, Taniai N, Bando K, Tajiri T: Transcatheter hepatic arterial embolization followed by microwave ablation for hemobilia from hepatocellular carcinoma. J Nippon Med Sch 2008, 75:284-288

23. Lin SZ, Tseng CW, Chen CC: Hepatic artery pseudoaneurysm presenting with Mirizzi syndrome and hemobilia. Clin Gastroenterol Hepatol 2009, 7:e73

24. Falkoff GE, Taylor KJ, Morse S: Hepatic artery pseudoaneurysm: diagnosis with real-time and pulsed Doppler US. Radiology 1986, 158:55-56.

25. Foley WD, Berland LL, Lawson TL, Maddison FE: Computed tomography in the demonstration of hepatic pseudoaneurysm with hemobilia. $J$ Comput Assist Tomogr 1980, 4:863-865.

26. Lee NK, Kim S, Lee JW, Lee SH, Kang DH, Kim DU, Kim GH, Seo HI: MR appearance of normal and abnormal bile: Correlation with imaging and endoscopic finding. Eur J Radiol 2009 in press.

27. Katz MC, Meng CH: Angiographic evaluation of traumatic intrahepatic pseudoaneurysm and hemobilia. Radiology 1970, 94:95-99.

28. Okazaki M, Ono H, Higashihara H, Koganemaru F, Nozaki Y, Hoashi T, Kimura T, Yamasaki S, Makuuchi M: Angiographic management of massive hemobilia due to iatrogenic trauma. Gastrointest Radiol 1991, 16:205-214

\section{Pre-publication history}

The pre-publication history for this paper can be accessed here: http://www biomedcentral com/1471-230X/10/81/prepub

doi: 10.1186/1471-230X-10-81

Cite this article as: Lee et al., Life-threatening hemobilia caused by hepatic pseudoaneurysm after T-tube choledochostomy: report of a case BMC Gastroenterology 2010, 10:81 